\title{
Assessing US Registered Dietitians Knowledge and Perceptions of a Gluten-Free Diet: A Mixed Methods Approach
}

Robert G. Brannan ( $\nabla$ brannan@ohio.edu )

Ohio University

Hannah Helton

Ohio University

Elizabeth A. Beverly

Ohio University

Deborah Murray

Ohio University

Melissa Russell

Ohio University

\section{Research Article}

Keywords: gluten, dietitian, gluten-free, mixed methods

Posted Date: March 3rd, 2021

DOI: https://doi.org/10.21203/rs.3.rs-267644/v1

License: (c) (i) This work is licensed under a Creative Commons Attribution 4.0 International License. Read Full License 


\section{Abstract}

\section{Background}

The practice of gluten-free diets is on the rise, evidenced by the increase in gluten-free sales from \$2.8 billion in 2015 to a projected $\$ 7.6$ billion in 2020. People with celiac disease and non-celiac gluten sensitivity are required to avoid gluten containing products. It is important that registered dietitians are knowledgeable about this topic due to the prevalence and popularity of the gluten-free diet by those with or without celiac disease or non-celiac gluten sensitivity.

\section{Methods}

A mixed methods approach using qualitative (focus group) was used to generate topics for a quantitative (survey) that assessed knowledge and perceptions of a gluten-free diet from a representative sample of 508 registered dietitians.

\section{Results}

Over $18 \%$ of respondents incorrectly identified gluten as a carbohydrate. Additionally, less than $80 \%$ of RDs correctly identified the presence or absence of gluten in semolina, spelt, kamut, and buckwheat. Regarding perceptions of a gluten-free diet, participants reported that the public is not aware of what gluten is, a gluten-free diet is not appropriate for the general public or weight loss, and the gluten-free diet is a fad when used outside of celiac disease and nonceliac gluten sensitivity.

\section{Conclusions}

There is a need for improved education of a gluten-free diet in the areas of sources of gluten, labeling, and gluten-related disorders among Registered Dietitians.

\section{Background}

Gluten is a storage protein found in the endosperm of wheat grains that supports the germination and development of the plant. Similar storage proteins are found in other grains, including barley and rye, and thus it is often stated that gluten is found in these grains as well (1). Gluten is a complex mixture of several proteins, the most common of which are glutenin and gliadin (2). These proteins are heat stable, and, with the combination of water and agitation, are responsible for many desirable effects in food science, including the formation of air pockets and moisture retention. As such, its properties are used extensively in baked goods, and it is commonly used as a thickener, emulsifier, gelling agent, or filler in many processed food products (3).

Some individuals are required to avoid gluten containing products due to a medical condition, such as the autoimmune condition celiac disease, wheat allergy, or non-celiac gluten sensitivity (4). However, these conditions are rare, and the prevalence of these conditions has remained relatively stable since 2009 (5). 
Thus, the increase in demand for gluten-free products has been attributed to the increasing prevalence of individuals choosing to avoid gluten for perceived benefits such as weight loss, or the perception that gluten is "bad for you," or unhealthy (5). A survey conducted by the NPD group indicated that almost a third of American adults would prefer to limit or completely avoid intake of gluten (6).

Successful elimination of dietary exposure to gluten requires careful attention to food labels to ensure a given product is gluten-free. The Codex Alimentarius of the Food and Agriculture Organization of the United Nations (FAO) and the World Health Organization (WHO) defines gluten-free products as those in which the total gluten does not exceed $20 \mathrm{mg} / \mathrm{kg}$ (7). Similarly, in August 2013, the U.S. Food and Drug Administration (FDA) issued a regulation mandating foods must contain less than 20 parts per million (ppm) in order to claim to be "gluten-free," "free of gluten," "no gluten," or "without gluten" (8). These regulations apply to all products except for meat, poultry, some egg products, and most alcoholic beverages, and was expanded in August 2020 to include hydrolyzed and fermented products. There is currently no mandated design or format for these claims on food packages; as such, not all products display these claims prominently.

Current labeling of gluten-free products is often an obstacle to adherence to a gluten-free diet (9). Purchasing gluten-free foods can be more challenging than merely looking for gluten-containing foods like wheat, barley, or rye on the ingredients list because gluten is not listed as a separate ingredient on food declarations and purchasers may be unfamiliar with gluten-containing ingredients such as malt vinegar or spelt. Consequently, many consumers mistakenly consume products with gluten that they assume to be gluten-free $(10,11)$. Unintentional exposure to gluten for those following a gluten-free diet for medical reasons could exacerbate the individual's disease symptoms (12).

As such, Registered Dietitian Nutritionists (RDN) must be equipped with sufficient knowledge about a gluten-free diet in order to provide optimal care to patients with celiac disease and non-celiac gluten sensitivity (1). Furthermore, with gluten-free sales increasing from \$2.8 billion in 2015 to a projected $\$ 7.6$ billion in 2020 (13), it is likely that sufficient knowledge of gluten and gluten-free diets will be required to enable RDN to provide education to individuals interested in a gluten-free diet for perceived health benefits, but without a medical condition requiring it (14). Thus, the objective of this study was to employ a mixed-methods approach to obtain and evaluate knowledge and perceptions of gluten and a gluten-free diet among RDN. Results of this study will be used to determine the level of need for further education on gluten and the gluten-free diet in the field and may yield valuable insight for the improvement of current educational materials.

\section{Methods}

All aspects of this research were approved by the Ohio University Institutional Review Board for the protection of human subjects in research in accordance with institutional guidelines. All participants agreed to informed consent indicating their acceptance of the minimal risks connected with the study.

\section{Focus groups}


Three focus groups were conducted to acquire insight into the knowledge and perceptions of gluten and gluten-free and included 26 individuals who participated. Participants were recruited from the local community via an email invitation and were excluded if they were not in the nutrition field or actively studying nutrition. The investigators developed a template for the focus group discussion in advance which assured that responses about participants knowledge of gluten and impressions of gluten-free diets and products were solicited across all three focus groups. The three focus groups were conducted by an experienced moderator and were audio-recorded and later transcribed for thematic analysis.

Content analysis was performed using NVivo 11 software (QSR International, Victoria, Australia). A researcher used the software to perform a detailed transcript review that identified common topics which were assigned codes. The codes included, but were not limited to, gluten information, gluten-related disorders, public awareness, education, labeling, gluten-free diet, and quality concerns in gluten-free products. Two different members of the research team independently coded the transcripts and discrepancies were reviewed, discussed, and resolved through consensus among the team.

\section{Questionnaire Development}

The questionnaire was piloted by nine RDN who participated in the focus groups and were given the opportunity to provide feedback on potentially problematic questions. After the pilot feedback was incorporated into the questionnaire, a link to the survey was sent to a list of 5000 randomly generated emails of the overall registry of approximately 97,000 Registered Dietitians in the US provided by the Commission on Dietetic Registration. A reminder email was sent after 7 days and the survey was available for a 17-day period. Responses were considered usable if all fields were complete, which resulted in 508 usable questionnaires for a 10\% return rate. A summary of the demographic results can be viewed in Table 1. Answers to the question about participants state of residence were further categorized into one of four regions (Northeast, Midwest, South, and West) according to standard classifications by the U.S. Census Bureau (1994).

The questionnaire was composed of seven open-ended or multiple-choice questions related to gluten and celiac disease and fourteen statements to which participants were asked to assess their level of agreement on a 5-point Likert scale ( 1 = strongly disagree, 2 = disagree, 3 = neither agree nor disagree, $4=$ agree, 5 = strongly agree), which is reported on a -2 (strongly disagree) to 2 (strongly agree) scale.

Data from the survey questionnaire was analyzed statistically using SPSS version 20 for Windows (SPSS, Inc., Chicago, III., U.S.A.). Data from the questionnaire was analyzed to establish measures of central tendency of responses for each question and analysis of variance (ANOVA) was used to determine significant differences $(P<0.05)$, with means were separated using Duncan's multiple range test.

\section{Results And Discussion}


Three focus groups were conducted each consisting of five to nine individuals. Not all of the 26 participants were RDN but all were in the nutrition field. Analysis of the focus group discussions led to the emergence of following themes.

\section{Focus Group Theme: Gluten Information}

All participants were aware that gluten is a protein found in wheat, rye, and barley. Several participants could name other specific details about gluten, such as its digestibility and impact on structure and texture of food products. For example, when asked to provide a single word for gluten's function, almost all named "structure" and "texture." There was general agreement about the increasing importance of knowledge about gluten:

If you're going to work in a restaurant or you're going to be a dietitian you need to know about gluten and gluten-free products.

\section{Focus Group Theme: Quality of Gluten-Free Products}

Participants noted that the quality of homemade and commercial gluten-free products has improved and acknowledged an increasing use of convenience products that are gluten-free. However, there was consensus that there is room for further quality improvement in the areas of excess chewiness, denseness, and lack of structure, as noted:

I think there is still a perception that some foods don't taste as good when they're gluten-free.

Participants expressed concern that food companies might be tempted to add excess sugar and fat in order to improve the taste of gluten-free items, the addition of which can negatively affect consumer health $(15,16)$.

\section{Focus Group Theme: Gluten-Free Diets}

The discussion about gluten-free diets centered on whether the diet should feature naturally gluten-free products or specialty products designed to be gluten-free. There was consensus that the "best" glutenfree diet utilizes naturally gluten-free items, especially fruits and vegetables, but also other nutrient dense foods such as rice, quinoa, nuts, and legumes. This agrees with the current literature, which recommends that a gluten-free diet be supplemented with nutrient dense grains (1). However, focus group participants acknowledged the widespread use of convenience specialty products, i.e., products that typically contain gluten but have been reformulated to be gluten-free, rather than naturally gluten-free products. As noted by a participant:

I think that's a sign that people don't really know what foods gluten is in.

\section{Focus Group Theme: Gluten-Related Disorders}


Awareness and knowledge about celiac disease and non-celiac gluten sensitivity were topics that came up throughout the focus groups, which is not surprising because all of the participants were from the nutrition field. Participants asserted that these are treatable conditions, and as noted by one participant:

I think that needs to be hit home pretty hard that it (gluten) is not a toxin that some people seem to think it is.

Participants were aware that celiac disease is an autoimmune disorder in which gluten cannot be properly digested and that if gluten is consumed, patients will experience negative side effects. They agreed that the only treatment is a gluten-free diet, which is consistent with current recommendations $(1,17)$. Participants also noted that some individuals claim to experience negative side effects following consumption of gluten, despite not having been diagnosed with celiac disease and noted the inconsistency in the definition of the condition referred to as nonceliac gluten sensitivity. This agrees with the current literature, which typically identifies a patient as having non-celiac gluten sensitivity when the possibility of celiac disease and wheat allergy have been eliminated and symptoms are alleviated on a gluten-free diet (18).

\section{Focus Group Theme: Public Awareness of Gluten}

The belief of focus group participants concerning a lack of public awareness of gluten can be summed up by this participant's response:

I feel like people don't know what gluten is. I mean, we're just very uneducated as a society in my opinion.

Participants described a common misperception that gluten is a carbohydrate and confusion between a gluten-free diet and a low-carb diet. Participants believed that the public is receiving their nutrition information from noncredible sources and blamed misinformation from celebrities and social media. Participants expressed concern that the public believes a gluten-free diet can be used as a nutritional intervention for conditions other than celiac disease or non-celiac gluten sensitivity. They termed a glutenfree diet as a fad when used in circumstances other than celiac disease or non-celiac gluten sensitivity.

\section{Focus Group Theme: Gluten-Free Labeling}

Opinions that were sorted into the gluten-free labeling category stem from the importance that participants ascribed to labeling as a means to alleviate the public's lack of information about gluten and assist those new to a gluten-free diet. As noted by one participant:

Gluten-free is going to be around, I mean it's not going anywhere.

Participants stated that it would be much easier to find acceptable options for those following a glutenfree diet if a product has the words "gluten-free" on the package. However, there was debate about the use of gluten-free labels, mostly relating to the use of a gluten-free label on products that have always been gluten-free. In one of the focus groups, a robust discussion focused on guacamole, a naturally 
gluten-free product, because it would be accurate to label it as gluten-free. However, participants wondered if customers might think that some guacamole contains gluten and the labeled guacamole version was reformulated to be gluten-free. leading to consumer confusion. The focus group results seem to indicate the need for consumer education about food labels, which is supported by recent literature (1).

\section{Focus Group Impact on Questionnaire Development}

The focus group results indicated those in the nutrition field likely are aware of many key issues concerning gluten, but what could not be assessed qualitatively is the level of awareness across the profession. Focus group results informed nearly all of the topics that were included in the gluten-free questionnaire for RDN. Focus group results determined that it is important for RDs to be knowledgeable about gluten and gluten-related disorders, so specific questions were created to assess 1) knowledge of gluten in certain foods, 2) populations that should follow a gluten-free diet, and 3) clinically relevant information about celiac disease. Focus group results also informed the selection and inclusion of statements that assessed RDN attitudes towards consumer concerns about gluten-related disorders, gluten-free diets, gluten-free products and ingredients, and gluten-free labeling.

\section{Questionnaire Demographics and Basic Knowledge of Gluten}

Demographic characteristics of a representative sample of 508 US RDN are summarized in Table 1. Overall, participants skewed female (97\%) and white (94\%), but were evenly distributed across age, years of experience, region, and whether their practice includes celiac disease. Slightly more than half $(56 \%)$ had more education that the Bachelor's degree required for eligibility to become a RDN. Responses were received from all states.

The questionnaire assessed RDN basic knowledge of gluten, foods that contain gluten, and the function of gluten in foods. Most RDN (81\%) correctly stated that gluten is a protein, however, $19 \%$ incorrectly thought that gluten is a carbohydrate. There was no misconception about the presence of gluten in wheat because all RDN correctly identified wheat as a gluten-containing food (Table 2). The correct identification of the presence of gluten in other gluten-containing foods ranged from $89 \%-38 \%$ in the order of rye, barley, semolina, spelt, and kamut. The gluten was identified at a higher rate in foods that are more commonly consumed (wheat, rye, barley) than in those that are less commonly known (semolina, spelt, kamut). There did not appear to be a misconception about the absence of gluten in corn, brown rice, quinoa, teff, or amaranth, as the absence of gluten was correctly identified in these foods by $99 \%, 96 \%$, $96 \%, 89 \%$, and $88 \%$ of RDN, respectively. However, there may have been confusion about the absence of gluten in buckwheat, as the absence of gluten was correctly identified by only $58 \%$ of RDN. The confusion about buckwheat being gluten-free might be due to participants' assumption that buckwheat is a type of wheat.

Common responses or synonyms to the open-ended question "Using a single word, describe the function of gluten in baked goods" were categorized into one of five categories: structure/texture (50\%), elasticity $(25 \%)$, binding $(15 \%)$, leavening $(6 \%)$, or other $(4 \%)$. It is not surprising that three quarters of respondents 
chose a single word to describe gluten that was either structure, texture, or elasticity because gluten is often referred to as a viscoelastic mass that provides structure and texture to baked goods. There is significant research to support the claim that the texture and structure of gluten-free products are different than their gluten-containing counterparts (19-22). Focus group participants noted the quality issues that exist in many gluten-free products as potentially frustrating to those following a gluten-free diet.

Taken together, these results show the need for targeted continuing education on the most basic aspects of gluten and a gluten-free diet. RDN were proficient at describing the presence or absence of gluten in common foods. However, it is concerning that nearly 1 in 5 RDN were unaware that gluten is a protein and RDN were not as proficient at describing the presence or absence of gluten in less common foods. An improved working knowledge of gluten and its sources could enhance patient/client interactions when dealing with gluten-free diets.

\section{Gluten-Free Diet and Celiac Disease}

Current estimates place the prevalence of celiac disease in the US at $0.5-1.3 \%$ (23). This study revealed that RDN overestimated the prevalence of celiac disease. Three quarters of RDN responded that the prevalence of celiac disease in the US was greater than $2 \% ; 30 \%$ greater than $10 \%$, and $7 \%$ of RDN thought the prevalence of celiac disease was greater than $25 \%$ of the population. It seems likely that the increased popularity of the gluten-free diet has caused RDN to believe that the prevalence of celiac disease is much higher than it actually is.

The study also revealed that RDN have a strong understanding of the definition and diagnostic method for diagnosing celiac disease. Shown in Table 3, almost all RDN (99\%) identified celiac disease as a condition requiring a gluten-free diet, a claim that is supported by the Evidence Analysis Library of the Academy of Nutrition and Dietetics (24). The survey also revealed that $90 \%$ of RDN correctly identified celiac disease as an autoimmune condition and $80 \%$ selected the correct diagnostic method for celiac disease, an intestinal biopsy.

Of the other conditions that RDN identified as requiring a gluten-free diet, $72 \%$ identified non-celiac gluten sensitivity, for which a gluten-free diet has become the choice for treatment $(18,25), 50 \%$ identified wheat allergy, and many fewer identified irritable bowel syndrome (12\%), Crohn's disease (12\%), autism (10\%), and type 1 diabetes (4\%). Although treatment for wheat allergy involves the elimination of wheat from the diet, the treatment does not exclude other gluten-containing grains (26), so a wheat-free diet is not necessarily a gluten-free diet. There has been research investigating the effectiveness of a gluten-free diet as a treatment method for irritable bowel syndrome and Crohn's disease, but the results are not definitive $(27,28)$. Overall, RDs were aware that celiac disease and NCGS are the only conditions with substantial research to support the use of a gluten-free diet.

\section{Appraisal of Consumer Concerns About Gluten-Free Issues from Registered Dietitians}


RDN rated their agreement with 14 statements of general consumer concern regarding gluten and glutenfree diets. Although randomly presented to the RDN, the statements were thematically grouped into four categories: Issues about celiac disease and non-celiac gluten sensitivity, gluten-free diets, gluten-free products and ingredients, and gluten-free labelling (Fig. 1). ANOVA was performed to explore if the demographic differences of the RDN affected their agreement with these statements. With the exceptions noted below, there were few significant differences in the data, indicating relatively homogenous agreement among the RDN across the demographic categories.

RDN strongly agreed that people with celiac disease should have regular appointments with a RDN and that non-gluten sensitivity is recognized as a diagnosable condition (Fig. 1A). This finding is reflective of the current research on non-celiac gluten sensitivity as a legitimate medical condition $(18,29)$ in spite of the fact that there are no standardized diagnostic criteria. RDN strongly disagreed with the statement that people with celiac disease can consume small amounts of gluten (Fig. 1A). Current guidelines state that a completely gluten-free diet is virtually impossible due to trace amounts of gluten consumed through unintentional cross-contamination, and ingestion of less than $10-20 \mathrm{ppm}$ will likely not cause damage to those with celiac disease (17). However, unintentional ingestion of more than 10-20ppm of gluten by people with celiac disease can cause intestinal damage, further hindering their ability to absorb nutrients $(17,30)$. It is very likely that RDN were responding to the recommendation to avoid cross-contamination with gluten to protect the safety of those with celiac disease.

The survey revealed strong quantitative agreement with the focus group's qualitative assessment that there is public misinformation that a gluten-free diet can be used as a nutritional intervention for conditions other than celiac disease or non-celiac gluten sensitivity and the characterization of the glutenfree diet as a fad when used under these circumstances. Specifically, the survey showed that RDN strongly agree that the gluten-free diet is a fad diet when used outside of celiac disease or non-celiac gluten sensitivity and strongly disagree that a gluten-free diet is appropriate for the general population (Fig. 1B). Research has shown that of the population consuming gluten-free products, only $25 \%$ were diagnosed with celiac disease (31). RDN also strongly disagree that a gluten-free diet is effective for weight loss (Fig. 1B), which is supported by research suggesting that a gluten-free diet is not effective for weight loss (32). However, there is less agreement among RDN when it comes to the consumption of specialty gluten-free products. In the focus groups, participants acknowledged the widespread use of convenience specialty products but expressed preference for naturally gluten-free items. The questionnaire revealed only tepid disagreement with the statement that gluten-free diets should only contain naturally gluten-free foods (Fig. 1B), perhaps reflecting this acknowledgement.

With respect to gluten-free products and ingredients, RDN strongly disagree that the public knows what gluten is (Fig. 1C), which mirrors the opinion of focus group participants and reflects the fact that one out of five RDN incorrectly identified gluten as a carbohydrate, not a protein, supports this opinion. There was neither strong agreement or disagreement that specialty gluten-free products are nutritionally comparable to similar gluten-containing products or that there are enough gluten-free options on the market (Fig. 1C). Research has shown that the gluten-free market grew by 178\% from 2013 to 2016 (31) and more recently 
gluten-free sales increased from \$2.8 billion in 2015 to a projected \$7.6 billion in 2020 (13). This growth indicates that there is likely a large array of options for those following a gluten-free diet.

In the area of gluten-free labelling, focus group participants indicated that if "gluten-free" was stated on food packaging, it would be much easier to find acceptable gluten-free options, however, there was disagreement about whether to use gluten-free labels on products that have always been gluten-free (Fig. 1D). RDN strongly agree that gluten should be included in the allergen statement on a food label but neither agreed nor disagreed that naturally gluten-free foods should be labelled. The statement, "People following a gluten-free diet should ignore gluten-free labels and look at ingredient list" produced no general agreement across all RDN (Fig. 1D) but significant differences between age, years of experience, and whether the RDN practice includes celiac disease (Table 4). The implication of this question is whether a gluten-free label is enough information or whether people should be responsible for reading and understanding the actual ingredient list. Mean agreement values for RDN younger than 45 and those with correspondingly less experience, i.e. less than 20 years, were significantly higher than for those who were older than 65 and have more than 40 years of experience. This suggests that younger RDN place more importance on the actual ingredient label than very seasoned RDN and could be related to the training that newer RDN receive on the topic. Another interesting finding concerning this question showed a significant difference between RDN whose practice includes celiac disease and those whose does not. RDN whose practice includes celiac disease disagreed whereas RDN whose area of practice does not include celiac disease agreed that people following a gluten-free diet should ignore gluten free labels and focus on the ingredient list.

\section{Conclusions}

RDN are experts in the field of nutrition, but this has exposed several areas of concern for RDN pertaining to sources of gluten, labeling, and gluten-related disorders among. Some RDN lack knowledge about gluten's classification as a protein. Over $18 \%$ of respondents incorrectly identified gluten as a carbohydrate. The authors of this study agree that this should be foundational knowledge for an RDN. As stated in the focus groups, there is a misconception by the public that gluten is a carbohydrate, and this study indicates that RDN also may be influenced to believe that gluten is a carbohydrate. Additionally, less than $80 \%$ of RDN correctly identified the presence or absence of gluten in semolina, spelt, kamut, and buckwheat. If RDN are unable to correctly identify the presence of absence of gluten from a list of ingredients, it may be more difficult to rely on clients for identifying gluten in ingredient lists, as indicated by many RDN who suggest that people should ignore gluten-free labels and look directly at the ingredient list. Many RDN vastly overestimated the prevalence of celiac disease, which may undermine the credibility of RDN in this area. Most RDN identified celiac disease and NCGS as conditions requiring a gluten-free diet, but that fact that nearly 50\% RDN would recommend a gluten-free diet for those with a wheat allergy might be cause for alarm. Although a gluten-free diet is wheat-free, a wheat-free diet does not need to be gluten-free, potentially limiting the foods that can be consumed by this population and unnecessarily increasing the risk of unintentional nutritional deficits. 
Gluten-free labeling is a topic for which the mixed methods approach of this study was very revealing. A

gluten-free label may be helpful for those just beginning a gluten-free diet, however it consumers may be confused about whether the labelled product is naturally gluten-free or contained gluten and has now been reformulated to be gluten-free. The questionnaire revealed that most RDs think gluten should be included on the ingredient list along with the top 8 allergens. Further research investigating the potential benefits of gluten-free labeling would be advantageous. As this is a topic with many facets, research focused specifically to labeling would be beneficial.

Education for RDN about various aspects of the gluten-free diet would benefit the profession, as knowledgeable RDN are more likely to provide valuable education to patient/clients on a gluten-free diet. Education in the form of continuing professional education units (CPEUs) could be a viable option for improving the knowledge of gluten and a gluten-free diet to RDN and results of this study could guide the development of Continuing Professional Education Units.

\section{Declarations}

\section{Ethics approval and consent to participate}

All methods were carried out in accordance with Ohio University's the institutional guidelines. Ethical clearance was obtained from Ohio University's institutional review board (IRB), protocol\# 15-E-400. All focus group participants signed a written informed consent form. Informed consent was obtained for participants of the questionnaire via an IRB-approved online consent form.

\section{Availability of data and materials}

The datasets used during the current study are available from the corresponding author on reasonable request.

\section{Competing interests}

The authors declare no competing interests.

\section{Funding}

Not applicable

\section{Authors' contributions}

$\mathrm{HH}$ and $\mathrm{RB}$ collected data. $\mathrm{RB}, \mathrm{EB}$, and $\mathrm{DM}$ provided insight into data collection and analysis. $\mathrm{HH}$ and $\mathrm{RB}$ wrote the initial draft and MR was a major contributor to the final manuscript. All authors read and approved the final manuscript.

\section{Acknowledgements}


The authors appreciate the focus group participants and the RDN who took the time to participation in the study.

\section{References}

1. See JA, Kaukinen K, Makharia GK, Gibson PR, Murray JA. Practical insights into gluten-free diets. Nat Rev Gastroenterol Hepatol. 2015;12(10):580-91.

2. Shewry P. What Is Gluten-Why Is It Special? Front Nutr. 2019;6(101):1-10.

3. Biesiekierski J. What is gluten? Journal of Gastroenterology and Hepatology. 2017;32(S1):78-81.

4. Igbinedion SO, Ansari J, Vasikaran A. Non-celiac gluten sensitivity: All wheat attack is not celiac. World J Gastroenterol. 2017;23(40):7201-10.

5. Kim H-S, Patel KG, Orosz E. Time Trends in the Prevalence of Celiac Disease and Gluten-Free Diet in the US Population: Results From the National Health and Nutrition Examination Surveys 2009-2014. JAMA Intern Med. 2016;176(11):1716-7.

6. Diez-Sampedro A, Olenick M, Maltseva T, Flowers M. A gluten-free diet, not an appropriate choice without a medical diagnosis. Journal of nutrition and metabolism. 2019;2019.

7. Food and Agricultural Organization. Standard for Foods for Special Dietary Use for Persons Intolerant to Gluten Codex Stan 118-1979. World Health Organization;

8. US Food and Drug Administration. Food labeling: gluten-free labeling of foods. 2013.

9. Zysk W, Głąbska D, Guzek D. Role of Front-of-Package Gluten-Free Product Labeling in a PairMatched Study in Women with and without Celiac Disease on a Gluten-Free Diet. Nutrients. 2019;11(2).

10. Melini V, Melini F. Gluten-Free Diet: Gaps and Needs for a Healthier Diet. Nutrients. 2019;11(1).

11. Silvester JA, Weiten D, Graff LA, Walker D JR, D.R. Is it gluten-free? Relationship between self-reported gluten-free diet adherence and knowledge of gluten content of foods. Nutrition. 2016;32(7-8):77783.

12. Bascuñán KA, Vespa MC, Araya M. Celiac disease: understanding the gluten-free diet. European Journal of Nutrition. 2017;56:449-59.

13. Statista Research Department. U.S. Gluten-free Foods Market - Statistics \& Facts [Internet]. 2020 [cited 2021 Jan 29]. Available from: https://www.statista.com/topics/2067/gluten-free-foodsmarket/

14. Reilly NR. The Gluten-Free Diet: Recognizing Fact, Fiction, and Fad. The Journal of Pediatrics. 2016;175:206-10.

15. Pellegrinia N, Agostoni C. Nutritional aspects of gluten-free products. J Scie Food Agric. 2015;95(12):2380-5.

16. Vici G, Belli L, Biondi M, Polzonetti V. Gluten free diet and nutrient deficiencies: A review. Clin Nutr. 2016;35(6):1236-41. 
17. Rubio-Tapia A, Hill ID, Kelly CP, Calderwood AH, Murray JA. ACG clinical guidelines: Diagnosis and management of celiac disease. The American Journal of Gastroenterology. 2013;108:656-76.

18. Catassi C, Elli L, Bonaz B, Bouma G, Carroccio A, Castillejo G, et al. Diagnosis of Non-Celiac Gluten Sensitivity (NCGS): The Salerno Experts' Criteria. Nutrients. 2015;7(6):4966-77.

19. Alencar NMM, Steel CJ, Alvim ID, Morais EC, Bolini HMA. Addition of quinoa and amaranth flour in gluten-free breads: Temporal profile and instrumental analysis. LWT - Food Sci Technol. 2015;62(2):1011-8.

20. Goswami D, Gupta RK, Mridula D, Sharma M, Tyagi SK. Barnyard millet based muffins: Physical, textural and sensory properties. LWT - Food Sci Technol. 2015;64(1):374-80.

21. Inglett GE, Chen D, Liu SX. Physical properties of gluten-free sugar cookies made from amaranth-oat composites. LWT - Food Sci Technol. 2015;63(1):214-20.

22. Kaur M, Sandhu KS, Arora A, Sharma A. Gluten free biscuits prepared from buckwheat flour by incorporation of various gums: Physicochemical and sensory properties. LWT - Food Sci Technol. 2015;62(1):628-32.

23. Singh P, Arora A, Strand TA, Leffler DA, Catassi C, Green PH, et al. Global prevalence of celiac disease: systematic review and meta-analysis. Clinical Gastroenterology and Hepatology. 2018;16(6):823-36.

24. Academy of Nutrition and Dietetics. Evidence Analysis Library. 2009.

25. Elli L, Roncoroni L, Bardella MT. Non-celiac gluten sensitivity: Time for sifting the grain. World J Gastroentero. 2015;21(27):8221-6.

26. Pietzak M. Celiac disease, wheat allergy, and gluten sensitivity: when gluten free is not a fad. JPEN Parenter Enter. 2012;36(1 Suppl):68-75.

27. Ruepert L, Quartero AO, Wit NJ, Heijden GJ, Rubin G. Bulking agents, antispasmodics and antidepressants for the treatment of irritable bowel syndrome. Cochran DB Syst Rev. 2011;10(8):003460.

28. Cruchet S, Lucero Y, Cornejo V. Truths, myths and needs of special diets: Attentiondeficit/hyperactivity disorder, autism, non-celiac gluten sensitivity, and vegetarianism. Ann Nutr Metab. 2016;68(supplement 1):43-50.

29. Fasano A, Sapone A, Zevallos V, Schuppan D. Nonceliac gluten sensitivity. Gastroenterology. 2015;148(6):1195-204.

30. Kelly CP, Bai JC, Liu E, Leffler DA. Advances in diagnosis and management of celiac disease. Gastroenterology. 2015;148(6):1175-86.

31. Roberts W. Gluten-free Foods - US. Mintel Group Ltd; 2016.

32. Gaesser GA, Angadi SS. Gluten-free diet: Imprudent dietary advice for the general population? J Acad Nutr Diet. 2012;112(9):1330-3.

\section{Tables}


Table 1 Demographic characteristics of registered dietitians responding to a gluten-free questionnaire ( $\mathrm{n}$ $=508)$ 


\begin{tabular}{|c|c|c|c|}
\hline Characteristic & Response Categories & Frequency & Percentage \\
\hline \multicolumn{4}{|l|}{ Gender } \\
\hline & Female & 493 & 97 \\
\hline & Male & 15 & 3 \\
\hline \multicolumn{4}{|c|}{ Age range years } \\
\hline & $25-34$ & 147 & 28 \\
\hline & $35-44$ & 131 & 25 \\
\hline & $45-54$ & 94 & 18 \\
\hline & $55-64$ & 110 & 21 \\
\hline & 65 and over & 26 & 5 \\
\hline \multicolumn{4}{|l|}{ Race } \\
\hline & White & 478 & 94 \\
\hline & Hispanic or Latino & 7 & 1 \\
\hline & Black or African American & 6 & 1 \\
\hline & Asian/Pacific Islander & 7 & 1 \\
\hline & Other & 10 & 2 \\
\hline \multicolumn{4}{|l|}{ Region } \\
\hline & Northeast & 97 & 19 \\
\hline & Midwest & 144 & 28 \\
\hline & South & 174 & 34 \\
\hline & West & 93 & 18 \\
\hline \multicolumn{4}{|l|}{ Education } \\
\hline & Doctoral degree & 6 & 1 \\
\hline & Professional degree & 48 & 9 \\
\hline & Master's degree & 231 & 45 \\
\hline & Bachelor's degree & 223 & 43 \\
\hline \multicolumn{4}{|c|}{ Years as an RDN } \\
\hline & $0-4$ years & 34 & 6 \\
\hline & 5-9 years & 116 & 22 \\
\hline
\end{tabular}




\begin{tabular}{|llll|}
\hline $10-19$ years & 168 & 33 \\
& $20-29$ years & 97 & 19 \\
& $30-39$ years & 72 & 14 \\
\hline Diagnosed Disorder & $40+$ years & 20 & 3 \\
\hline & & & 29 \\
& Celiac disease & 15 & 51 \\
\hline Practice includes celiac disease & Nonceliac gluten sensitivity & 26 & 13 \\
& Wheat allergy & 7 & 50 \\
\hline & Yes & 254 & 50 \\
\hline & No & 254 & 50 \\
\hline
\end{tabular}

Table 2 Frequency of registered dietitians correctly identifying the presence of gluten in gluten-containing and the absence of gluten in gluten-free foods $(n=508)$

\begin{tabular}{|llllll|}
\hline $\begin{array}{l}\text { Foods that } \\
\text { Contain gluten }\end{array}$ & Frequency & Percentage & $\begin{array}{l}\text { Foods that do not } \\
\text { contain gluten }\end{array}$ & Frequency & Percentage \\
\hline Wheat & 508 & 100 & Corn & 502 & 99 \\
\hline Rye & 448 & 88 & Brown rice & 489 & 96 \\
\hline Barley & 442 & 87 & Quinoa & 486 & 96 \\
\hline Semolina & 365 & 72 & Teff & 453 & 89 \\
\hline Spelt & 263 & 52 & Amaranth & 448 & 88 \\
\hline Kamut & 191 & 38 & Buckwheat & 294 & 58 \\
\hline
\end{tabular}

Table 3 Frequency of registered dietitians identifying populations that should follow a gluten-free diet $(\mathrm{n}=$ 508) 


\begin{tabular}{|lll|}
\hline Population & Frequency & Percentage \\
\hline Celiac disease & 505 & 99 \\
\hline Nonceliac gluten sensitivity & 363 & 71 \\
\hline Wheat allergy & 253 & 49 \\
\hline Irritable bowel syndrome & 63 & 12 \\
\hline Crohn's disease & 63 & 12 \\
\hline Autism & 53 & 10 \\
\hline Type 1 diabetes & 20 & 3 \\
\hline
\end{tabular}

Table 4 Mean level of agreement on a 5-point Likert scale ( 1 = strongly disagree, 2 = disagree, $3=$ neither agree nor disagree, 4 = agree, $5=$ strongly agree) from registered dietitians $(n=508)$ to the question "People following a gluten-free diet should ignore gluten-free labels and just look at the ingredient list" based on three demographic characteristics. (Note: means with different superscripts within a demographic characteristic are significantly different at the $p$-value shown.) 


\begin{tabular}{|c|c|c|c|c|}
\hline Characteristic & Response Categories & Mean & $\mathbf{n}$ & p-value \\
\hline \multicolumn{5}{|c|}{ Age range years } \\
\hline & $25-34$ & $3.2^{\mathrm{a}}$ & 147 & 0.029 \\
\hline & $35-44$ & $3.0^{\mathrm{a}}$ & 131 & \\
\hline & $45-54$ & $2.9^{\mathrm{a}, \mathrm{b}}$ & 94 & \\
\hline & $55-64$ & $2.8^{\mathrm{a}, \mathrm{b}}$ & 110 & \\
\hline & 65 and over & $2.5^{\mathrm{b}}$ & 26 & \\
\hline \multicolumn{5}{|c|}{ Years as an RDN } \\
\hline & $0-4$ years & $3.4^{\mathrm{a}}$ & 34 & 0.013 \\
\hline & $5-9$ years & $3.1^{\mathrm{a}, \mathrm{b}}$ & 116 & \\
\hline & $10-19$ years & $3.1^{\mathrm{a}, \mathrm{b}}$ & 168 & \\
\hline & $20-29$ years & $2.8^{\mathrm{b}, \mathrm{c}}$ & 98 & \\
\hline & $30-39$ years & $2.8^{\mathrm{b}, \mathrm{c}}$ & 72 & \\
\hline & $40+$ years & $2.4^{\mathrm{c}}$ & 20 & \\
\hline \multicolumn{5}{|c|}{ Practice includes celiac disease } \\
\hline & Yes & $2.8^{b}$ & 254 & 0.016 \\
\hline & No & $3.1^{\mathrm{a}}$ & 254 & \\
\hline
\end{tabular}

Figures 


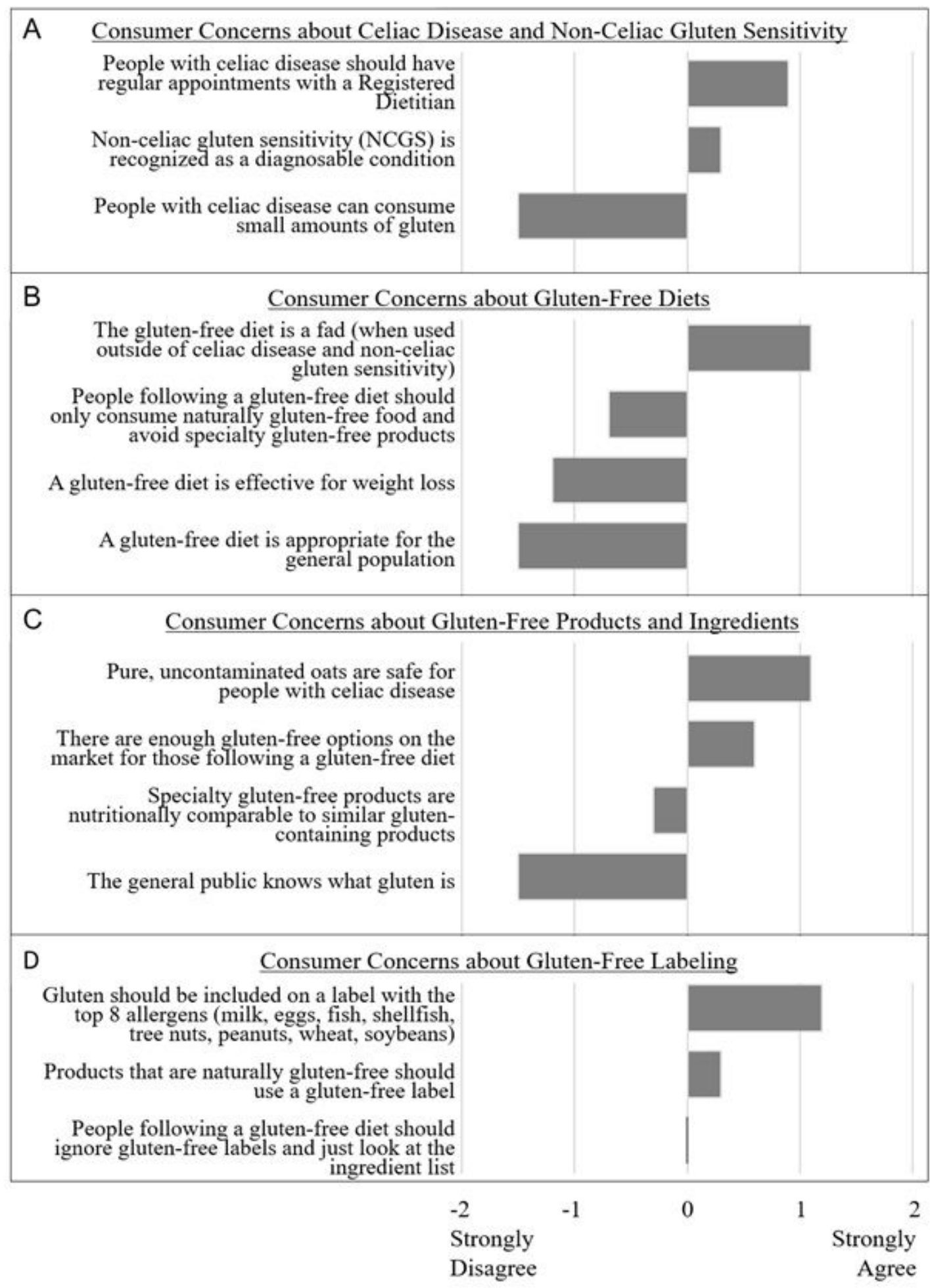

\section{Figure 1}

Responses about gluten-free issues from Registered Dietitians ( $n=508)$ using a 5-point Hedonic agreement scale (-2 = strongly disagree, 0 = neither agree nor disagree, 2 = strongly agree). 